\title{
A FULLERENE FORMATION MODEL PROPOSED FROM MOLECULAR DYNAMICS SIMULATIONS
}

\author{
Yasutaka Yamaguchi and Shigeo Maruyama \\ Department of Mechanical Engineering \\ The University of Tokyo, \\ 7-3-1 Hongo, \\ Bunkyo-ku, Tokyo 113 \\ Japan
}

\begin{abstract}
By using the molecular dynamics method, a clustering process of randomly distributed carbon atoms was simulated. A $\mathrm{C}_{60}$ imperfect fullerene obtained in the simulation was kept at $2500 \mathrm{~K}$ in order to evaluate the effect of the collision-free annealing process which was almost ignored in the clustering simulation. Through successive pentagon-migration transformations, the perfect fullerene structure was achieved within a plausible time scale of 200 ns. Similar annealing simulations for smaller precursor clusters were also performed to infer the annealing effect at each stage of clustering process. Based on these simulations, a new formation model of empty fullerene was proposed. Moreover, the formation process of metal containing fullerene was also simulated using virtual L-J metal atoms.
\end{abstract}

\section{INTRODUCTION}

The molecular dynamics method has been serving as a very useful tool for studies of microscale and molecular scale heat transfer problems. Those problems in the material processing such as in semiconductor industries usually include the chemical reaction process and the light-matter interaction, which are theoretically very challenging. Here, we report a possibility of the molecular dynamics simulation of chemical reaction problem in the generation process of an exciting new carbon material, fullerene discovered by Kroto et al. (1985). In addition to the original empty spherical $\mathrm{C}_{60}$ and $\mathrm{C}_{70}$, metal containing fullerene [Chai et al. (1991), Shinohara et al. (1992), Kikuchi et al. (1993), Takata et al. (1993)], higher fullerene (Kikuchi et al., 1992) and carbon nanotubes (Iijima, 1991) were successively produced and isolated. Recently, the high quality generation of single-wall nanotubes (SWNTs) (Thess et al., 1996) has demonstrated new possibilities of applications of this material. Although fullerene is now recognized as an attractive new material, the formation mechanism of such symmetric hollow caged structure is still unknown, because the generation techniques of fullerene [Krätschmer et al. (1990), Haufler et al.
(1991)] were discovered almost accidentally. Besides physical interests, it is important to understand the formation mechanism in order to find the optimum condition of generating metal containing fullerene, higher fullerene and SWNT.

Typical fullerene formation models based on experimental insights are summarized in Fig. 1. These are

(1) "Pentagon road" model proposed by Haufler et al. (1991),

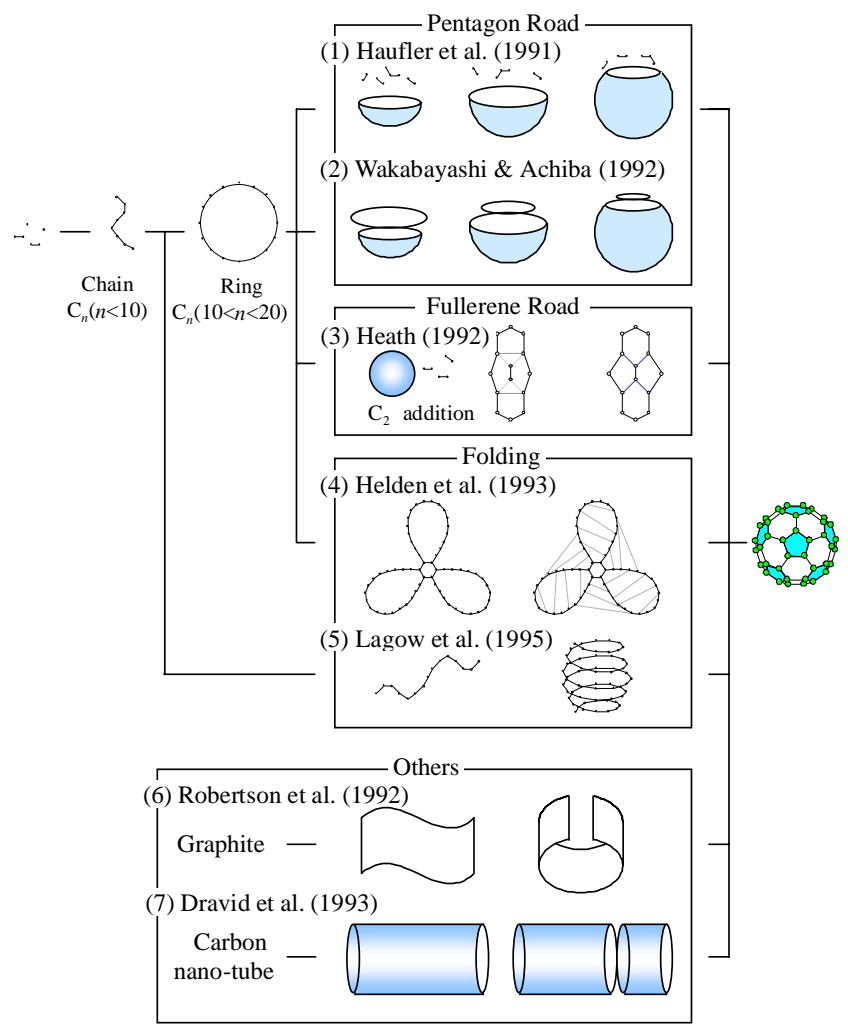

Fig. 1 Fullerene formation models 
explained that additions of pentagonal rings into the hexagonal network were the primary reason for the curvature leading to a spherical structure. They discussed that the inclusion of pentagonal rings had advantage by reducing the number of dangling bonds. In addition, they suggested that it was unlikely to have two pentagons next to each other because they would induce too much stress: known as IPR (Isolated Pentagon Rule).

(2) Wakabayashi \& Achiba (1992) suggested a similar model that fullerene was constructed by stacking of proper sized carbon rings.

(3) Heath (1992) proposed "fullerene road" model that clusters grew as linear chains up to $\mathrm{C}_{10}$, rings for $\mathrm{C}_{10}$ to $\mathrm{C}_{20}$, fullerene from about $\mathrm{C}_{30}$, and successive $\mathrm{C}_{2}$ additions at the point of two neighboring pentagons followed until the satisfaction of the IPR. This is the reversed process of laser dissociating experiments.

(4) Helden et al. (1993) and Hunter et al. (1993) showed experimental results using the ion-chromatography that the isomer structure of $\mathrm{C}_{60}{ }^{+}$bi-cyclic or tri-cyclic ring annealed to fullerene $\mathrm{C}_{60}$ under high temperature condition, and suggested these structures as precursors of fullerene.

(5) Lagow et al. (1995) suggested a similar model that a long chain would anneal to the fullerene via a spiral structure.

(6) Robertson et al. (1992) simulated the curling process of a graphite sheet by the molecular dynamics method.

(7) Dravid et al. (1993) speculated that fullerene was made from chips of carbon nanotubes.

Experimental results using a mixture of ${ }^{12} \mathrm{C}$ and ${ }^{13} \mathrm{C}$ isotopes showed that the graphite material was once decomposed into small pieces such as atoms, dimers or trimers [Meijer \& Bethune (1990), Hawkins et al. (1991), Ebbesen et al. (1992)]. In contrast, another experimental result using naphthalene as the combustion resource showed the magic numbers of fullerene indicating the original carbon number of naphthalene (Taylor et al., 1993). That meant the complete decomposition itself was not an inevitable process.

We have performed molecular dynamics simulations of the clustering process of carbon atoms to investigate the fullerene formation mechanism, and the temperature dependence of the cluster structures was observed [Yamaguchi \& Maruyama (1997), Maruyama \& Yamaguchi (1997)]; resulted in graphitelike structures when the control temperature $T_{c}$ was lower than $2500 \mathrm{~K}$, fullerene-like caged structures for $2500 \mathrm{~K}<T_{c}<3500$ $\mathrm{K}$, chaotic 3-dimensional structures for $T_{c}>3500 \mathrm{~K}$. In addition, the clustering process under lower density condition at $T_{c}=3000 \mathrm{~K}$ was calculated in order to give much longer collision free time - though much shorter than that in practice [Fig. 2] (Maruyama \& Yamaguchi, 1997). Typical clusters shown in the bottom panel of Fig. 2 were simple chain or ring structures for $\mathrm{C}_{n}(n<20)$, tangled poly-cyclic structures for around $\mathrm{C}_{n}(20<n<30)$, random caged structure for $\mathrm{C}_{n}(n>30)$, $\mathrm{C}_{60}$ and $\mathrm{C}_{70}$ with hollow caged structure.

Although $\mathrm{C}_{60}$ and $\mathrm{C}_{70}$ were actually obtained, they were not the perfect fullerene satisfying the IPR. The highly compressed annealing process seemed to be the problem. In this paper, we speculated the possibility of rearrangement into the perfect fullerene by giving sufficient collision-free annealing time, and examined the preferred structures of precursor clusters in the same fashion. Based on these results, we presented a new formation model of empty fullerene. In addition, another molecular dynamics simulation of the formation of metal containing fullerene was achieved, and the difference in the formation mechanism was discussed.

\section{METHOD}

The potential function among carbon atoms (Brenner, 1990) was the same as our previous reports [Yamaguchi \& Maruyama (1997), Maruyama \& Yamaguchi (1997)]. The total potential energy $E_{b}$ was expressed as the sum of binding energy as follows.

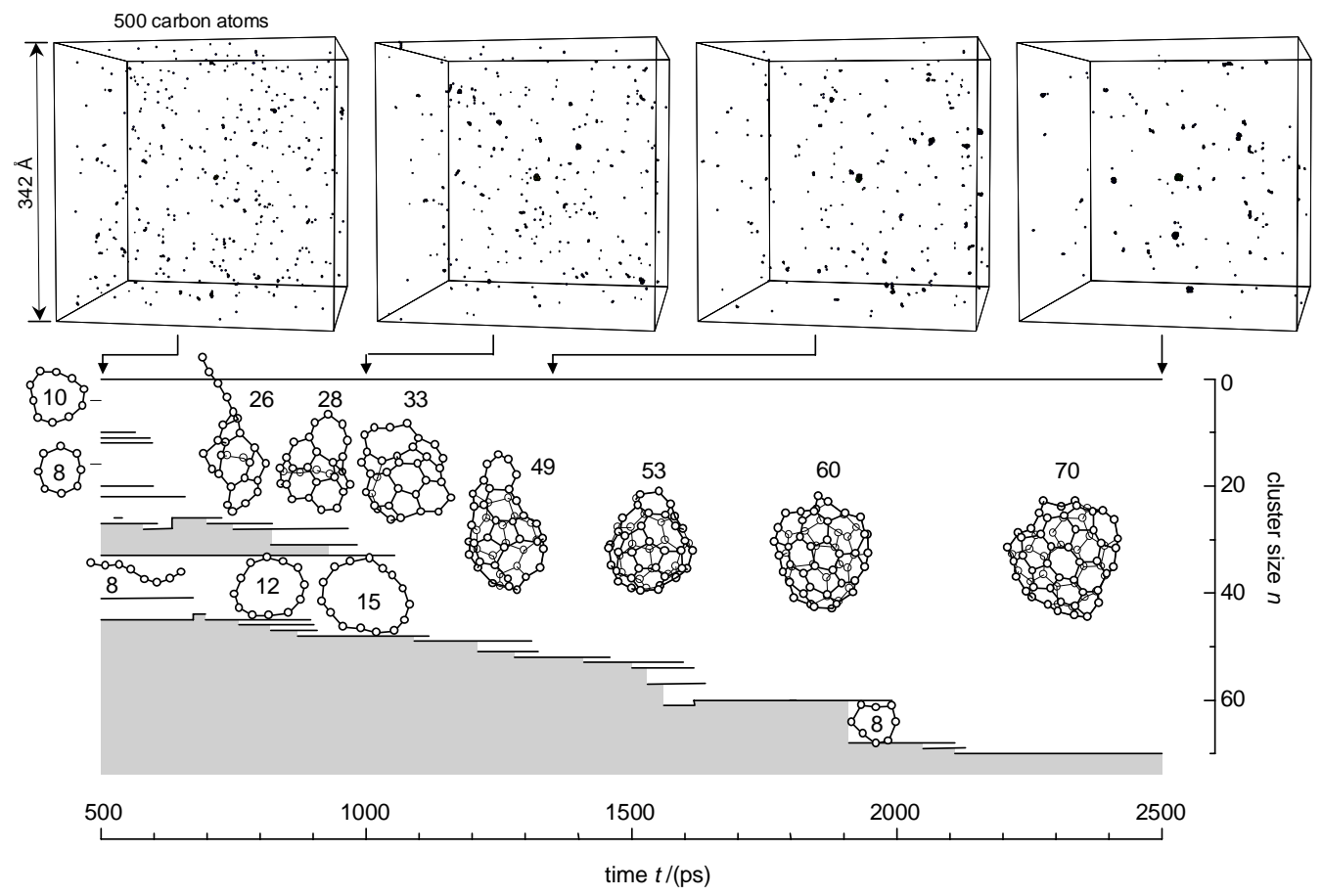

Fig. 2 Snapshots of the clustering process and a dynamic path to a caged cluster $\mathrm{C}_{70}$. 
TABLE 1

Potential Parameters

\begin{tabular}{cccccccccc}
\hline$D_{e} /(\mathrm{eV})$ & $S$ & $\beta /(1 / \AA)$ & $R_{e} /(\AA)$ & $R_{1} /(\AA)$ & $R_{2} /(\AA)$ & $\delta$ & $a_{0}$ & $c_{0}$ & $d_{0}$ \\
\hline 6.325 & 1.29 & 1.5 & 1.315 & 1.7 & 2.0 & 0.80469 & 0.011304 & 19 & 2.5 \\
\hline
\end{tabular}

$$
\begin{aligned}
E_{b} & =\sum_{i} \sum_{j(i>j)}\left[V_{R}\left(r_{i j}\right)-B^{*}{ }_{i j} V_{A}\left(r_{i j}\right)\right] \\
V_{R}(r) & =f(r) \frac{D_{e}}{S-1} \exp \left\{-\beta \sqrt{2 S}\left(r-R_{e}\right)\right\} \\
V_{A}(r) & =f(r) \frac{D_{e} S}{S-1} \exp \left\{-\beta \sqrt{2 / S}\left(r-R_{e}\right)\right\}
\end{aligned}
$$

Where $V_{R}(r)$ and $V_{A}(r)$ were repulsive and attractive terms, respectively, described as the function of the distance $r$ between atoms $i$ and $j$, and both terms included the cut-off function $f(r)$. The additional term $B^{*}{ }_{i j}$ was combined to the attractive term as the function of the angle $\theta_{i j k}$ between bond $i-j$ and $i-k$ expressed as follows.

$$
\begin{gathered}
B_{i j}^{*}=\frac{B_{i j}+B_{j i}}{2}, \quad B_{i j}=\left(1+\sum_{k(\neq i, j)}\left[G_{c}\left(\theta_{i j k}\right) f\left(r_{i k}\right)\right]\right)^{-\delta} \\
G_{c}(\theta)=a_{0}\left(1+\frac{c_{0}{ }^{2}}{d_{0}{ }^{2}}-\frac{c_{0}{ }^{2}}{d_{0}{ }^{2}+(1+\cos \theta)^{2}}\right)
\end{gathered}
$$

Parameters are shown in TABLE 1.

The temperature control method was also the same as our previous report [Yamaguchi \& Maruyama (1997), Maruyama \& Yamaguchi (1997)]. The motion of a cluster was divided into translational, rotational and vibrational motions, and the system temperatures $T_{T}, T_{R}$ and $T_{V}$ were defined as the total average of each motion in the system. Then, simple velocity scaling was independently enforced to each motion every $0.1 \mathrm{ps}$ so that the difference between the control temperature $T_{c}$ and each temperature was reduced to $60 \%$. Verlet's method was adopted to integrate the equation of motion.

\section{MOLECULAR DYNAMICS SIMULATIONS Annealing Process to the Perfect Fullerene}

In order to evaluate the effect of the collision-free annealing, the $\mathrm{C}_{60}$ cluster originally obtained in our simulation [Fig. 2] (Maruyama \& Yamaguchi, 1997) was kept under high temperature condition of $T_{c}=2500 \mathrm{~K}$ by removing all other clusters to collide with. Transformations of the network structure are shown in Fig. 3, where the Schlegel network diagram is adopted: e. g. the 3-dimensional initial structure (parenthesized) is expressed as the diagram above. Although the initial cluster had hollow caged structure, it contained 4 atoms having dangling bonds (empty symbol), neighboring pentagons (shaded) and 6 heptagons, and was quite different from the perfect fullerene $\mathrm{C}_{60}$ consists of 20 hexagons and 12 isolated pentagons. The bottom panel of Fig.3 shows the averaged potential energy per atom $E_{P}$ and the number of dangling bonds $N_{D B}$ during the annealing process. Here the instantaneous dangling bonds disappeared within 50 ps were omitted. The network structure changed frequently to reduce the strain, with seldom chances of generating dangling bonds which corresponded to higher potential energy. After about 215 $\mathrm{ns}$, the dangling bond did not appear, and the migrations of pentagons lead the structure to the perfect icosahedral fullerene $\mathrm{C}_{60}$ as shown in the top sequences of Fig. 3. After $221.7 \mathrm{~ns}$, the perfect $\mathrm{C}_{60}$ structure was conserved for a long time until 234.8 ns. In the whole process after $215 \mathrm{~ns}$, the cluster rearranged the

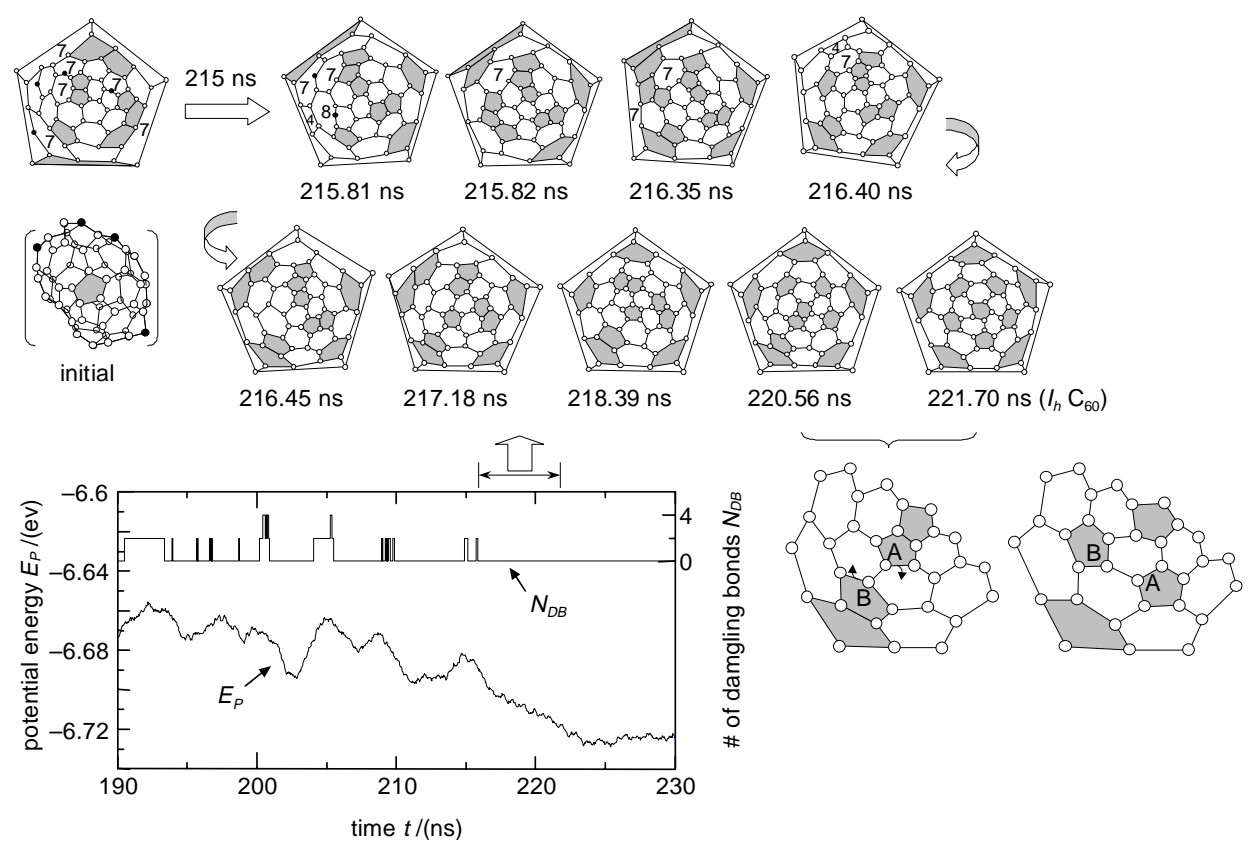

Fig. 3 Annealing process to the perfect fullerene $C_{60}$. 
network structure toward lower potential energy, and almost all the transformations can be regarded as the organized rearrangement called the Stone-Wales transformation (Stone \& Wales, 1986): two atoms between pentagons (atom A and B in Fig. 3) rotates 90 degrees to change the relative positions of pentagons. In addition, $\mathrm{C}_{70}$ perfect structure was also achieved in about 150 ns from the $C_{70}$ caged cluster in Fig. 2 by the same annealing simulation.

These are the first demonstration of self assemble of perfect $\mathrm{C}_{60}$ and $\mathrm{C}_{70}$ structures by molecular simulations.

In practice, experimental annealing temperature can be estimated to be about $1000 \sim 1500 \mathrm{~K}$ for the laser irradiation method (Haufler et al., 1991) and $1000 \mathrm{~K}$ for the arc discharge method (Maruyama et al., 1995). The imposed higher temperature condition of $2500 \mathrm{~K}$ in this simulation seems inappropriate. However, if we assume that the S-W transformation is the main reaction in the caged cluster, the time and temperature scale can reasonably agree with the experimental conditions; from the viewpoint of Arrhenius's reaction rate, the number of reactions in $200 \mathrm{~ns}$ at $2500 \mathrm{~K}$ corresponds to that in $0.5 \mathrm{~ms}$ at $1500 \mathrm{~K}$ and in $10 \mathrm{~s}$ at $1000 \mathrm{~K}$ with the estimated activation energy of $2.5 \mathrm{eV}$ in this simulation (Maruyama \& Yamaguchi, 1997). This rough extrapolation is very dangerous because it is based on the assumption that the S-W transformation is the only possible reaction regardless of temperature. This assumption can be allowed here because of the large difference between the chemical energy scale and the temperature energy scale. In a sense, this is similar to the concept of multiple time step calculations, and the careful usage of this technique must help to compensate for the time scale problem of general molecular dynamics studies related to the energy transfer between chemical and thermal energy.

\section{Dynamic Structures of Precursor Clusters}

It is obviously unfair to give sufficient annealing time only for the final structures $\mathrm{C}_{60}$ and $\mathrm{C}_{70}$. Here, the smaller precursors as $\mathrm{C}_{26}, \mathrm{C}_{33}$ and $\mathrm{C}_{48}$ were picked up from the simulation of Fig. 2 and isolated under high temperature condition of $T_{c}=3000 \mathrm{~K}$ without collisions. Fig. 4 shows typical structures, number of bonds $\left(N_{B}\right)$ and potential energy $\left(E_{P}\right)$ per atom. For $\mathrm{C}_{26}$ cluster [Fig. 4 (a)], flat structure was preferred to tangled poly-cyclic structure. As shown in the bottom diagram, the flat structures were energetically more stable. At that size, the tangled structures would induce too much stress and the number of bonds was almost the same for both structures. On the other hand, the $\mathrm{C}_{33}$ cluster [Fig. 4 (b)] changed back and forth between flat and random caged structures. At this size, the opportunity to choose these two structures seemed to be the same, though random cage was energetically more stable due to the reduction of dangling bonds. Then, the choice of energetically unfavored flat structure was possible because of the entropy effect at this temperature. There were a large variety of flat structures compared to the unique condensed random cage structure.

Here, we should note that when the clustering simulation was performed under lower temperature conditions, the initial flat structure could not overcome the energy barrier to transform to the random cage structure (Yamaguchi \& Maruyama, 1997). On the other hand, under high temperature condition, the chaotic 3-dimensional structure was chosen for further randomness (Yamaguchi \& Maruyama, 1997).

\section{FORMATION MODEL OF EMPTY FULLERENE}

In addition to our previous clustering simulations summarized in Fig. 2, the perfect fullerene was achieved from imperfect fullerene and precursors at each stage were analyzed. Considering these results and the temperature dependence of the cluster structure (Yamaguchi \& Maruyama, 1997), we propose a new fullerene formation model as in Fig. 5.

By laser irradiation or arc discharge method, the graphite source is once decomposed into atoms or dimers. Upon cooling process, these carbon clusters grow larger from the simple structures like chain or ring for $\mathrm{C}_{n}(n<20)$, and flat structures for around $\mathrm{C}_{n}(20<n<30)$. Then, the cluster meets the turning point at around $\mathrm{C}_{30}$; if the temperature is too low, it grows larger keeping the flat structure, while if the temperature is too high, the tangled poly-cyclic structure is favored. Only when an

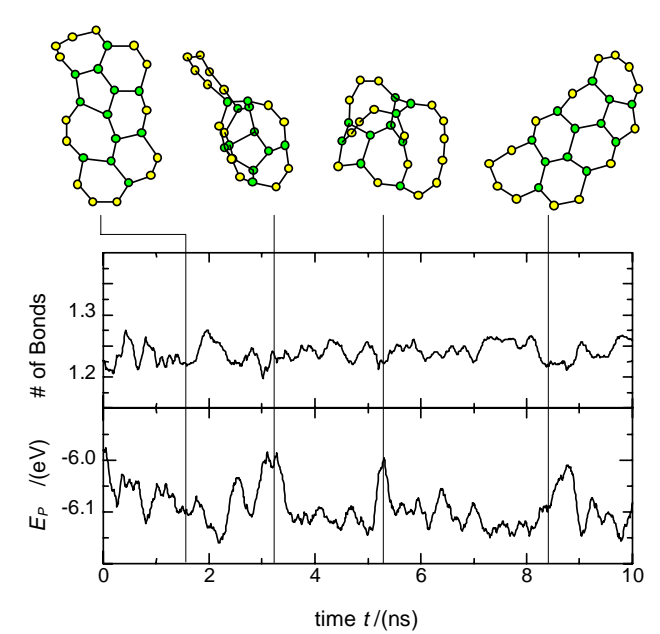

(a) $\mathrm{C}_{26}$ cluster favored flat structures compared to tangled poly-cyclic structures.

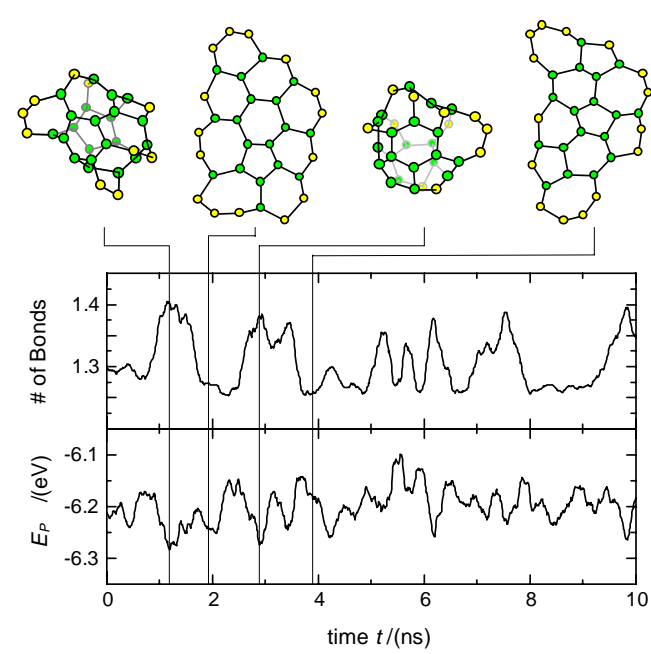

(b) $\mathrm{C}_{33}$ cluster equally favored flat and random cage structures.

Fig. 4 Dynamic structures of smaller precursor clusters at $T_{c}=3000 \mathrm{~K}$. 


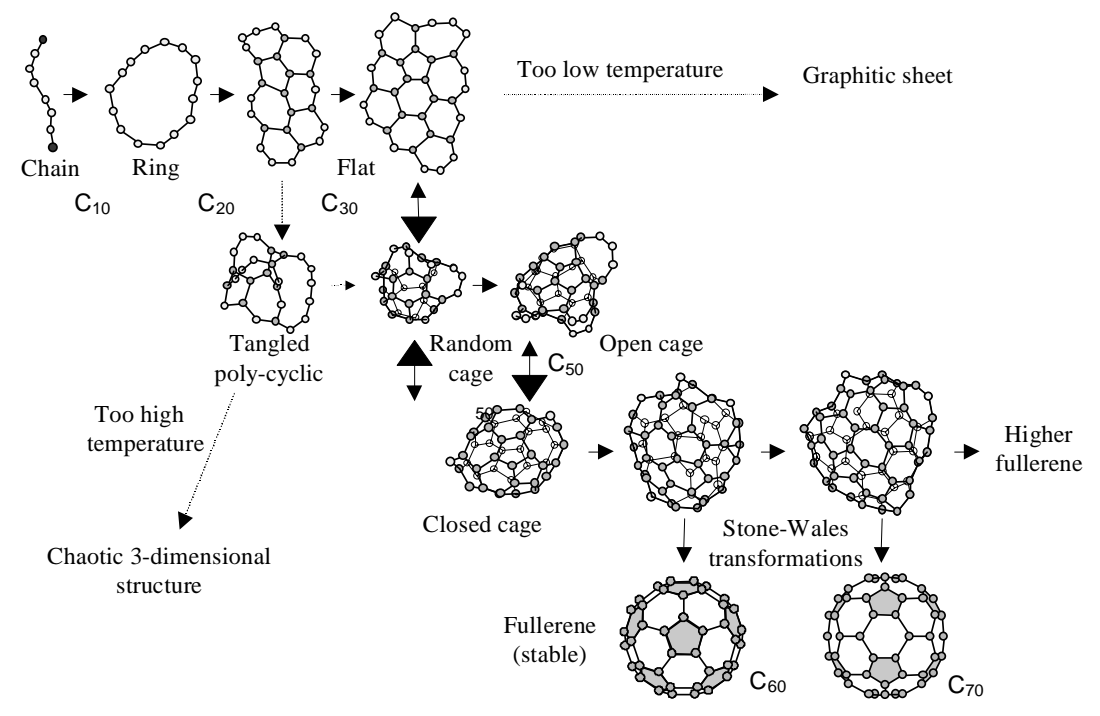

Fig. 5 Present fullerene formation model.

adequate temperature condition or cooling rate is applied, they can be rearranged to the hollow random caged structure. However, at that size, the number of carbon atoms is not enough to form a closed cage, and a lot of dangling bonds remain in the structure. Then, it grows larger with rearranging the network structure during the collision free interval due to its small collisional cross section, and finally reaches the perfect fullerene $\mathrm{C}_{60}$ satisfying the IPR. If it fails to become $\mathrm{C}_{60}$ by inappropriate addition, $\mathrm{C}_{70}$ will be the second candidate. Assuming that the perfect fullerene satisfying the IPR is not reactive to further collisions, $\mathrm{C}_{60}$ can be the most probable fullerene to stop the growth.

The structures from $\mathrm{C}_{20}$ to $\mathrm{C}_{40}$ in our model do not look so sophisticated as the precursors in the "pentagon road" model [Haufler et al. (1991), Wakabayashi \& Achiba (1992)]. The whole process is similar to the "pentagon road" model of Heath (1992), however, we propose the meta-stable random cage structure in which all atoms can have three bonds. This will overcome the problem of Fullerene road; unlikely assumption of $\mathrm{C}_{2}$ addition to the correct position between pentagons next to each other. In addition, our model does not contradict to the experimental results of Helden et al. (1993) and Hunter et al. (1993) where the tri-cyclic structured $\mathrm{C}_{60}$ was assumed to be the precursor, and so as the experimental result using naphthalene (Taylor et al., 1993).

\section{FORMATION OF METAL CONTAINING FULLERENE}

Although metal containing fullerene (metallofullerene) has been expected to be another attractive material, it is not open to applications because of the lack of the sample. In practice, the yield of metallofullerene is less than $0.1 \%$ by present generation techniques. The establishment of the efficient

\section{TABLE 2}

\section{L-J Potential Parameters}

\begin{tabular}{ccc}
\hline & $\varepsilon /(\mathrm{eV})$ & $\sigma /(\AA)$ \\
\hline metal-carbon & 0.6290 & 3.267 \\
metal-metal & 1.648 & 3.164 \\
\hline
\end{tabular}

generation technique is a very challenging task.

We have tried another molecular dynamics simulation for metallofullerene to speculate a key feature of the formation process. As the first step, both metal-metal and metal-carbon potentials were assumed to have the simple Lennard-Jones form as follows.

$$
E=4 \varepsilon\left\{(\sigma / r)^{12}-(\sigma / r)^{6}\right\}
$$

Where $r$ denoted the distance between atoms. Potential parameters $\varepsilon$ and $\sigma$ were determined as in TABLE 2, based on the ionic radius and cohesive energy for Yttrium.

Adding another 5 metal atoms in the initial cell of the simulation as in Fig. 2, the clustering process was calculated at $T_{c}=3000 \mathrm{~K}$. Fig. 6 shows the growing process of a metal containing fullerene. A ring structure $\mathrm{C}_{9}$ with a metal atom coalesced with a $\mathrm{C}_{32}$ cluster at about $2200 \mathrm{ps}$ and formed an open cap structure $\mathrm{C}_{43}$ with the metal atom attached in the center of the open edge. After growing to the size of $\mathrm{C}_{46}$ without closing the cap, another $\mathrm{C}_{16}$ cluster came at about 2750 ps and the metal atom was completely confined in the center of $\mathrm{C}_{63}$ cage, and grew further with rearranging the structure.

It should be noted that compared to the whole process of 2.5 ns in Fig. 2, the attachment of a metal atom happened at the very late stage of $2 \mathrm{~ns}$ in Fig. 6. This is comparable to the experimental observation that carbon is a unique material which can make clusters at very high temperature. With a metal, the open cap structure was preferred because the number of carbon atoms was too small to close to the cage, hence, the structure was more similar to that in the "pentagon road" model. However, since the attachment of metal atoms could happen only at the very late stage, additions of small clusters like $\mathrm{C}_{1}$ or $\mathrm{C}_{2}$ could not be expected. Probably, this is the reason of the low yield of metal containing fullerene. Moreover, it is very probable to have another metal atom on the cap because the metal atom is exposed at the open edge for a long time. Actually, 4 other metal atoms in this simulation made dimers before enclosed in the caged carbon network. This result corresponds to the experimental results that the yield of metallofullerene drops when more than about $1 \%$ of metal is 


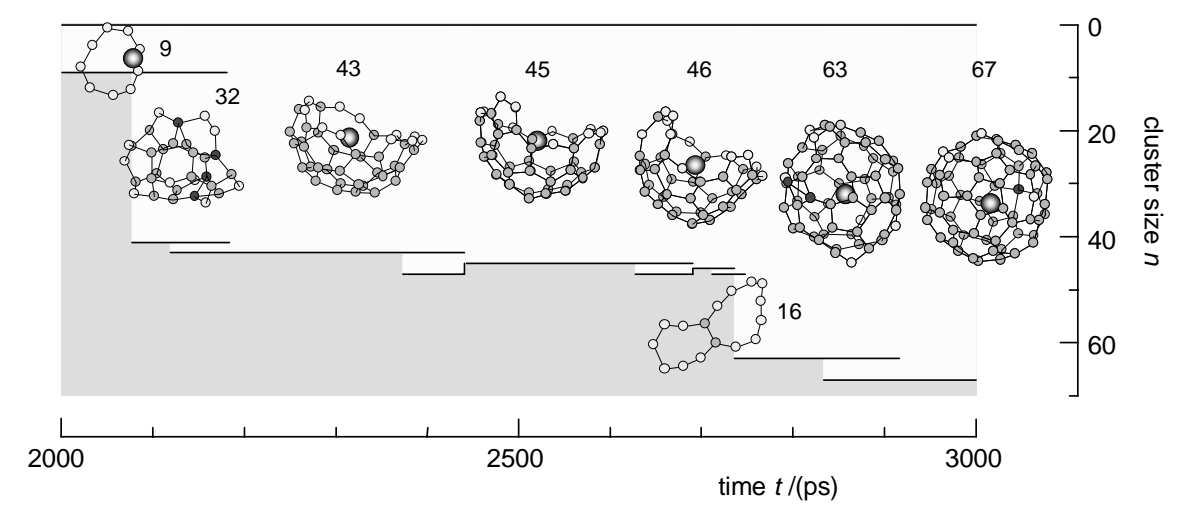

Fig. 6 Growing process of a metallofullerene.

added.

\section{CONCLUSIONS}

The annealing process of a $\mathrm{C}_{60}$ cluster was simulated from the initial structure obtained in the clustering simulation from isolated carbon atoms. The perfect fullerene $\mathrm{C}_{60}$ structure was achieved through spontaneous transformations under reasonable time and temperature condition. The preferred dynamic structures of small precursor clusters were also studied. Considering these results, a new fullerene formation model was proposed and compared with reported models. The formation process of metal containing fullerene was also discussed from a simulation using virtual metal atoms.

\section{ACKNOWLEDGEMENTS}

This work was supported by Grant-in-Aid for JSPS Fellows (No.08004746) from the Ministry of Education, Science, Sports and Culture, Japan.

\section{REFERENCES}

Brenner, D. W., 1990, Empirical Potential for Hydrocarbons for Use in Simulating the Chemical Vapor Deposition of Diamond Films, Phys. Rev. B, vol. 42, pp.9458-9471.

Chai, Y. et al., 1991, Fullerenes with Metals Inside, J. Phys. Chem., vol. 95, pp.7564-7568.

Dravid, V. P. et al., 1993, Buckytubes and Derivatives: Their Growth and Implications for Buckyball Formation, Science, vol. 259, pp.1601-1604.

Ebbesen T. W. et al., 1992, The Mechanistics of Fullerene Formation, Chem. Phys. Letters, vol. 191, pp. 336-338.

Haufler, R. E. et al., 1991, Carbon Arc Generation of $\mathrm{C}_{60}$, Proc. Mat. Res. Soc. Symp., 206, pp.627-638.

Hawkins, J.M. et al., 1991, Statistical Incorporation of ${ }^{13} \mathrm{C}_{2}$ Units into $\mathrm{C}_{60}$, J. Am. Chem. Soc., vol.113, pp.9394-9395.

Heath, J. R., 1992, Synthesis of $\mathrm{C}_{60}$ from Small Carbon Clusters, A Model Based on Experiment and Theory, Fullerenes, pp.1-23, American Chemical Society, Washington, D. C.

Helden, G. et al., 1993, Experimental Evidence for the Formation of Fullerenes by Collisional Heating of Carbon Rings in the Gas phase, Nature, 363, pp.60-63.

Hunter, J. et al., 1993, Annealing $\mathrm{C}_{60}{ }^{+}$: Synthesis of Fullerenes and Large Carbon Rings, Science, vol. 260, pp.784786.
Iijima, S., 1991, Helical Microtubules of Graphitic Carbon, Nature, vol. 354, pp.56-58.

Kikuchi, K. et al., 1993, Isolation and Characterization of the Metallofullerene $\mathrm{LaC}_{82}$, Chem. Phys. Lett., vol. 216, pp.23-26.

Kikuchi, K. et al., 1992, Isolation and Identification of Fullerene Family: $\mathrm{C}_{76}, \mathrm{C}_{78}, \mathrm{C}_{82}, \mathrm{C}_{84}, \mathrm{C}_{90}$ and $\mathrm{C}_{96}$, Chem. Phys. Lett., 188, pp.177-180.

Krätschmer, W. et al, 1990, Solid $\mathrm{C}_{60}$ : a New Form of Carbon, Nature, 347, pp.354-358.

Kroto, H. W. et al., 1985, $\mathrm{C}_{60}$ : Buckminsterfullerene, Nature, vol. 318, pp.162-163.

Lagow, R. J. et al., 1995, Synthesis of Linear Acetylenic Carbon: The "sp" Carbon Allotrope, Science, vol. 267, pp.362367.

Maruyama, S. et. al., 1995, Temperature Field Measurement during the Arc-discharge Fullerene Generation Process, Proc. 32nd National Heat Transfer Conf., vol. 2 ,pp.569-570.

Maruyama, S. \& Yamaguchi, Y., 1997, Molecular Dynamics of the Formation Process of Fullerene (2nd Report, Annealing to the Perfect $\mathrm{C}_{60}$ Structure), Trans. JSME, vol. 63-611B, pp. 2405-2412.

Meijer, G. \& Bethune, D.S., 1990, Laser Deposition of Carbon Clusters on Surfaces: A New Approach to the Study of Fullerene, J. Chem. Phys., vol. 93, pp.7800-7802.

Robertson, D. H. et al., 1992, On the Way to Fullerenes: Molecular Dynamics Study of the Curling and Closure of Graphitic Ribbons, J. Phys. Chem., vol. 96, pp.6133-6135.

Shinohara, H. et. al, 1992, Mass Spectroscopic and ESR Characterization of Soluble Yttrium-Containing Metallofullerenes $\mathrm{YC}_{82}$ and $\mathrm{Y}_{2} \mathrm{C}_{82}$, J. Phys. Chem., vol. 96, pp.3571-3573.

Takata, M. et al., 1995, Confirmation by X-ray Diffraction of the Endohedral Nature of the Metallofullerene $\mathrm{Y} @ \mathrm{C}_{82}$, Nature, vol. 377, pp.46-48.

Taylor, R. et al., 1993, Formation of $\mathrm{C}_{60}$ by Pyrolysis of Naphthalene, Nature, vol. 366, pp.728-731.

Thess, A. et al., 1996, Crystalline Ropes of Metallic Carbon Nanotubes, Science, vol. 273, pp.483-487.

Wakabayashi, T. and Achiba, Y., 1992, A Model for the $\mathrm{C}_{60}$ And $C_{70}$ Growth Mechanism", Chem. Phys. Letters, vol. 190, pp.465-468.

Yamaguchi, Y. \& Maruyama, S., 1997, Molecular Dynamics of the Formation Process of Fullerene (1st Report, The formation of a Caged Structure and Controlled Temperature), Trans.JSME, vol. 63-611B, pp.2398-2404. 